\title{
A Multiplicative Noise Removal Approach Based on Partial Differential Equation Model
}

\author{
Bo Chen, Jin-Lin Cai, Wen-Sheng Chen, and Yan Li \\ College of Mathematics and Computational Science, Shenzhen University, Shenzhen 518060, China \\ Correspondence should be addressed to Wen-Sheng Chen, chenws@szu.edu.cn
}

Received 26 February 2012; Accepted 23 March 2012

Academic Editor: Ming Li

Copyright ( $) 2012$ Bo Chen et al. This is an open access article distributed under the Creative Commons Attribution License, which permits unrestricted use, distribution, and reproduction in any medium, provided the original work is properly cited.

\begin{abstract}
Multiplicative noise, also known as speckle noise, is signal dependent and difficult to remove. Based on a fourth-order PDE model, this paper proposes a novel approach to remove the multiplicative noise on images. In practice, Fourier transform and logarithm strategy are utilized on the noisy image to convert the convolutional noise into additive noise, so that the noise can be removed by using the traditional additive noise removal algorithm in frequency domain. For noise removal, a new fourth-order PDE model is developed, which avoids the blocky effects produced by secondorder PDE model and attains better edge-preserve ability. The performance of the proposed method has been evaluated on the images with both additive and multiplicative noise. Compared with some traditional methods, experimental results show that the proposed method obtains superior performance on different PSNR values and visual quality.
\end{abstract}

\section{Introduction}

Image denoising plays an important role in the areas of image processing. A real recorded image may be distorted by many expected or unexpected random factors, of which random noise is an unavoidable one [1,2]. The objective of image denoising or filtering is to recover the true image from the noisy one. One of the challenges during the denoising process is to preserve and enhance the important features. For images, edge is one of the most universal and crucial features. Denoising via linear filters normally does not give satisfactory performance since both noises and edges contain high frequencies. Therefore, some nonlinear filters [3-18] have been proposed. Median filter [1] is one of the classical examples. Wavelet-based image filters [19-22] are developing quickly. PDE-based nonlinear diffusion filters [23-26] also make a hit on image denoising. One of PDE-based methods is the famous total variation model (TVM) [27-35]. TVM has been improved in theory and algorithm continuously. 
Recently, Kim [23] proposed a model called $\alpha \beta \omega(\mathrm{ABO})$-model by hybridizing a nonconvex variant of the TVM, the motion by mean curvature (MMC) [29], and Perona-Malik model [4] to deal with the mixture of the impulse and Gaussian noises reliably. In [23], they apply the essentially nondissipative difference $(\mathrm{ENoD})$ schemes $[5,6]$ for the MMC component to eliminate the impulse noise with a minimum (ideally no) introduction of dissipation. Many denoising methods are also employed in medical image processing [36-39].

Due to the coherent nature of some complicated image acquisition processes, such as ultrasound imaging, synthetic aperture radar (SAR) and sonar (SAS), and laser imaging, the standard additive noise model, so prevalent in image processing, is inadequate. Instead, multiplicative noise models, that is, in which the noise field is multiplied by (not added to) the original image, provide an accurate description of coherent imaging systems [40-42]. Multiplicative noise is naturally dependent on the image data. Various adaptive filters [43,44] for multiplicative noise removal have been proposed. Experiments have shown that filtering methods work well when the multiplicative noise is weak.

In this paper, a new fourth-order PDE model is introduced by improving the original fourth-order PDE model [24] in order to get high fidelity of the denoised images. To solve the model efficiently and reliably, we suggest a simple and symmetrical difference schemes. Median filter is exploited to alleviate the speckle effects in the processed image. At the same time, a new multiplicative noise removal algorithm based on fourth-order PDE model is proposed for the restoration of noisy image. To apply the proposed model for removal of multiplicative noise, the Fourier transform is used to change convolution into product; meanwhile, the logarithmic transformation is used to convert multiplicative noise into additive one. Experimental results show that the proposed method gets nice result in restoring images, especially in edge preservation and enhancement.

The rest of this paper is organized as follows. In Section 2 we investigate a general model of multiplicative noise. Total variation model and its discretization are introduced in Section 3. In order to avoid the blocky effects of second-order PDE model and preserve edges, a new fourth-order PDE denoising model is proposed in Section 4. Section 5 is devoted to a study of multiplicative noise removal method, and an algorithm based on fourth-order PDE model is developed. Numerical results are presented in Section 6. We summarize our conclusions in Section 7.

\section{Multiplicative Noise Model}

Noise removal or reduction is very important in image processing community. The objection of image denoising or filtering is to recover the true image from a noisy one. There are different noise types in real world. Multiplicative noise is common beside additive noise. Quality of images may degenerate while images' obtaining, transferring, and storage. The movement of objects, the defects of the imaging system, the noise of the inherent record equipment, and external disturbance also cause the image noise. Under the assumption that imaging system is linear translation invariance system, we can use the following degradation model to describe the multiplicative noise images:

$$
u_{0}(x, y)=h_{d}(x, y) * f(x, y)+n(x, y)
$$




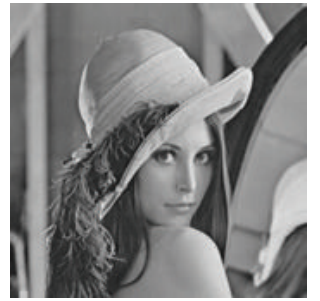

(a)

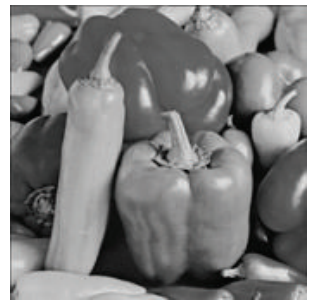

(e)

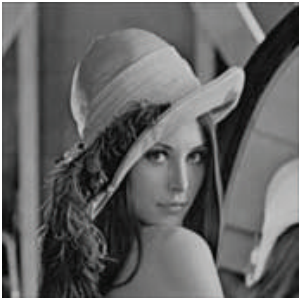

(b)

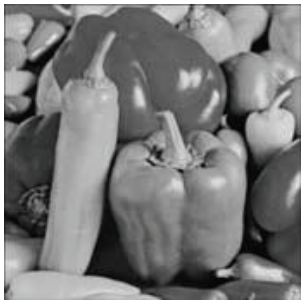

(f)

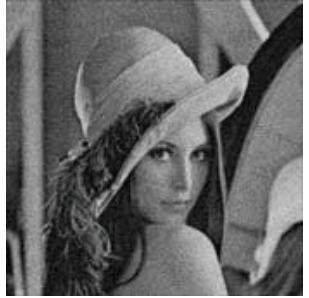

(c)

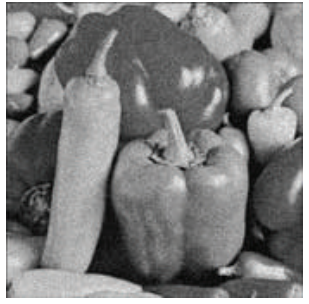

(g)

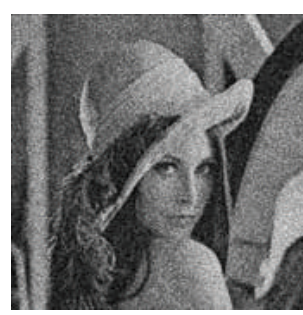

(d)

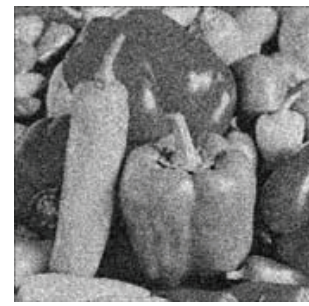

(h)

Figure 1: Multiplicative noise images. (a) Original "Lena" image; (b) image convolution with template $3 \times$ 3 of (a); (c) image with Gaussian white noise of mean 0 and variance 0.01 ; (d) image with Gaussian white noise of mean 0 and variance 0.05 ; (e) original "vegetables" image; (f) image convolution with template $3 \times 3$ of $(\mathrm{a})$; (g) image with Gaussian white noise of mean 0 and variance 0.01 ; (h) image with Gaussian white noise of mean 0 and variance 0.05 .

where $f$ is the ideal image, $u_{0}$ is the noised image, $n$ denotes the additive noise with mean 0 and variance $\sigma^{2}$, ${ }^{*}$ denotes convolution operation, $h_{d}$ denotes the point spread function (PSF), andGaussian function can be considered as one of the classical PSF:

$$
G_{d}(x, y)=\exp \left\{\frac{-\left(x^{2}+y^{2}\right)}{2 \sigma^{2}}\right\}
$$

Therefore, the synthesized images with multiplicative noise in this paper are generated for ideal images convolution with 2D Gaussian kernels and then noised with additive Gaussian white noise. An example is shown in Figure $1 . h_{d}$ in (2.1) is chosen as (2.2), namely Gaussian function templates. $3 \times 3$ Gaussian function template $T_{3}=(1 / 16) \times\left[\begin{array}{lll}1 & 2 & 1 \\ 2 & 4 & 2 \\ 1 & 2 & 1\end{array}\right]$ is employed here.

\section{Total Variation Model}

In order to recover the true image $f$ as much as possible and/or to find a new image $u$ in which the information of interest such as object boundary in the image is more obvious and/ or more easily extracted, we will discuss PDE-based image denoising in this section. Secondorder PDE models have been studied as a useful tool for image denoising. The classical model of them is total variation model (TVM) [27], and we will introduce it.

TVM was first proposed by Rudin et al. [27]. It is now one of the most successful tools in image restoration. TVM has a simple fixed filter structure. In terms of the mathematical foundation, unlike most statistical filters, TVM is based on functional analysis and geometry. 


$$
\begin{gathered}
(i-1, j) \\
\uparrow \\
(i, j-1) \leftarrow(i, j) \rightarrow(i, j+1) \\
\downarrow \\
(i+1, j)
\end{gathered}
$$

Figure 2: Four neighboring image structure.

The additive noise removal problem is converted to energy function minimization problem as below:

$$
\begin{aligned}
& u=\operatorname{argmin}\left\{E_{\mathrm{TV}}(u)\right\} \\
& E_{\mathrm{TV}}(u)=\iint_{\Omega}|\nabla u| d x d y+\frac{\lambda}{2} \iint_{\Omega}\left(u_{0}-u\right)^{2} d x d y,
\end{aligned}
$$

where $\Omega$ denotes image domain, and $\lambda$ is Lagrange multiplier. The selection of the parameter $\lambda$ is very important for the smoothing result. The corresponding Euler-Lagrange equation is

$$
-\nabla \cdot\left(\frac{\nabla u}{|\nabla u|}\right)+\lambda\left(u-u_{0}\right)=0
$$

and the steepest descent marching gives

$$
\frac{\partial u}{\partial t}=\nabla \cdot\left(\frac{\nabla u}{|\nabla u|}\right)-\lambda\left(u-u_{0}\right)
$$

To avoid singularities in flat regions or at local extreme, $|\nabla u|$ in (3.2) is regularized to $|\nabla u|_{\varepsilon}=\sqrt{|\nabla u|^{2}+\varepsilon^{2}}$ for a small positive parameter $\varepsilon$. Chan et al. [30] deduce discrete iterative equation of TV model as follows:

$$
u_{\alpha}^{n+1}=\sum_{\beta \in N(\alpha)} h_{\alpha \beta} u_{\beta}^{n}+h_{\alpha \alpha} u_{\alpha}^{0}
$$

where $u_{\alpha}^{0}$ denotes the pixel value at node $\alpha$ in the noisy image, $n$ denotes iteration times, $u_{\alpha}^{n}$ denotes the image pixel value after $n+1$ iterations, $N(\alpha)$ denotes field of node $\alpha$ (see Figure 2). The filter coefficients $h_{\alpha \alpha}$ and $h_{\alpha \beta}$ are given by

$$
\begin{aligned}
& h_{\alpha \alpha}=\frac{\lambda}{\lambda+\sum_{\beta \in N(\alpha)} w_{\alpha \beta}}, \\
& h_{\alpha \beta}=\frac{w_{\alpha \beta}}{\lambda+\sum_{\beta \in N(\alpha)} w_{\alpha \beta}},
\end{aligned}
$$




$$
\begin{gathered}
w_{\alpha \beta}=\frac{g_{\alpha}^{n}+g_{\beta}^{n}}{2 h^{2}}, \\
g_{\alpha}^{n}=\frac{1}{\left|\nabla u_{\alpha}^{n}\right|^{\prime}} \\
\left|\nabla u_{\alpha}^{n}\right| \approx \sqrt{\frac{1}{2 h^{2}} \sum_{\beta \in N(\alpha)}\left(u_{\beta}^{n}-u_{\alpha}^{n}\right)^{2}} .
\end{gathered}
$$

Here, for any node $\alpha h_{\alpha \alpha}+\sum_{\beta \in N(\alpha)} h_{\alpha \beta}=1$.

In conclusion, TV denoising algorithm steps can be summarized as follows:

(1) to assign parameter $\lambda$ and $a$;

(2) compute the local variation $\left|\nabla u_{\alpha}^{n}\right|$ by (3.7);

(3) compute respectively $g_{\alpha}^{n}$ and $w_{\alpha \beta}^{n}(3.6)$;

(4) compute the filter coefficients $h_{\alpha \alpha}$ and $h_{\alpha \beta}$ by (3.5);

(5) calculate iterative equation (3.4).

For TV filtering process, the computational cost can be reduced by the algorithm. TVM not only can remove noise but also can keep the image edge information. Some experimental results are shown in Figure 3. TVM is better than the traditional denoising methods not only in PSNR values but also in visual quality.

\section{A New Fourth-Order PDE Denoising Model}

In order to avoid the blocky effects (seen in Figure 3(f) and Figure 4(f)) widely seen in images processed by anisotropic diffusion while preserve edges, You and Kaveh [24] proposed a fourth-order PDE for noise removal. Motivated by [24] and TVM, we proposed a novel model in $[25,26]$. The new approach combines the advantages of the famous TVM and original fourth-order PDE model. It can avoid the blocky effects and get high fidelity (improve the quality of the processed image), which is important for image filter application (see Figure 4).

Consider the energy function as follows:

$$
E(u)=\iint_{\Omega}\left(f\left(\left|\nabla^{2} u\right|\right)+\frac{\lambda}{2}\left|u-u^{0}\right|^{2}\right) d x d y,
$$

where $\Omega$ is the image domain and $\lambda>0$ is a parameter similar as in TVM. $u^{0}$ is the noisy image. $\nabla^{2}$ denotes Laplacian operator and we require $f$ is an increasing function and bigger than zero. Therefore, the minimization of the functional is equivalent to smoothing the image as measured by $\left|\nabla^{2} u\right|$.

The corresponding Euler-Lagrange equation is

$$
\nabla^{2}\left(f^{\prime}\left(\left|\nabla^{2} u\right|\right) \operatorname{sign}\left(\nabla^{2} u\right)\right)+\lambda \cdot\left(u-u^{0}\right)=0
$$




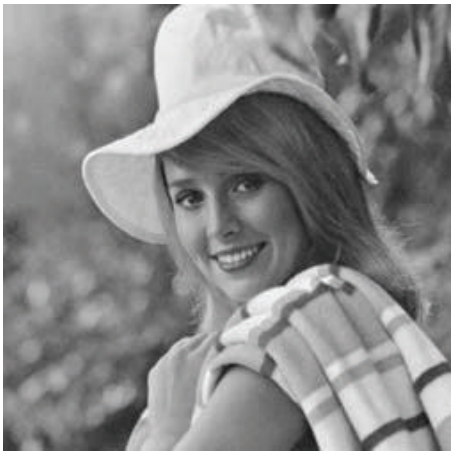

(a)

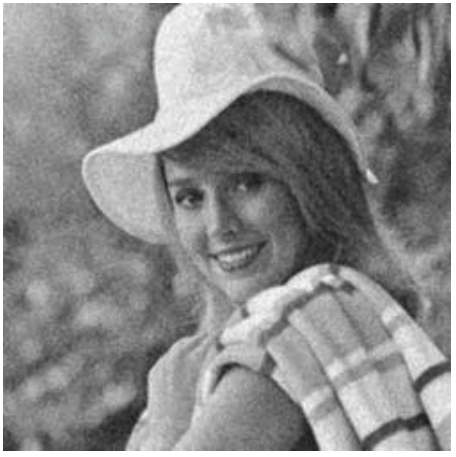

(d)

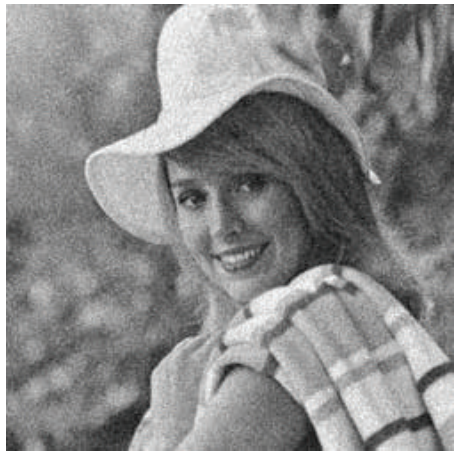

(b)

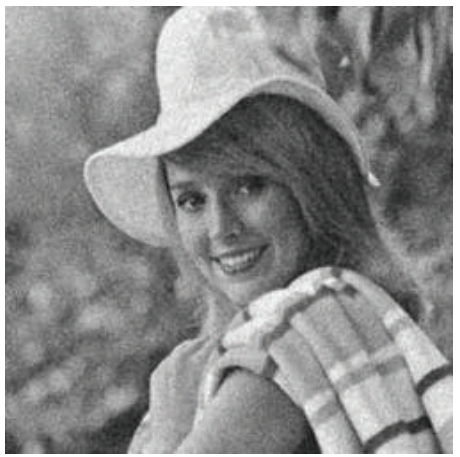

(e)

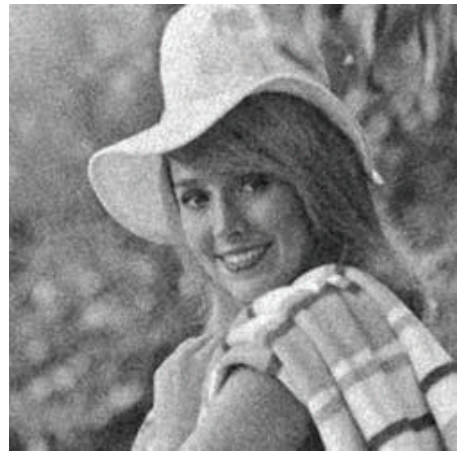

(c)

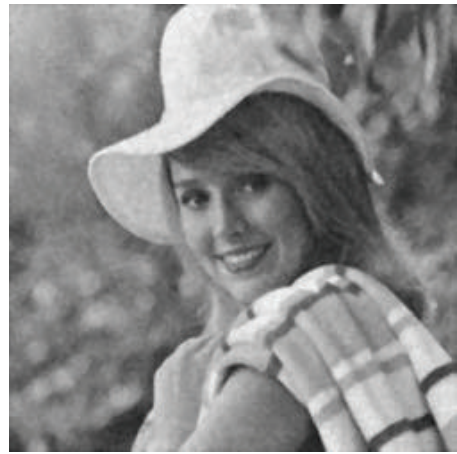

(f)

Figure 3: Detail of restoring noisy Elaine image $(512 \times 512)$ with different filters. (a) Original image; (b) noisy image, PSNR = 20.0742; (c) median, PSNR = 26.4554; (d) averaging, PSNR = 27.6876; (e) wiener, PSNR = 26.7866; (f) TVM, PSNR = 30.0919.

where sign is the signed distance function, so (4.2) can be written as

$$
\nabla^{2}\left(f^{\prime}\left(\left|\nabla^{2} u\right|\right) \frac{\nabla^{2} u}{\left|\nabla^{2} u\right|}\right)+\lambda \cdot\left(u-u^{0}\right)=0
$$

If we define $c(s)=f^{\prime}(s) / s$, which is

$$
\nabla^{2}\left(c\left(\left|\nabla^{2} u\right|\right) \nabla^{2} u\right)+\lambda \cdot\left(u-u^{0}\right)=0
$$

therefore, the Euler equation may be solved through the following gradient descent procedure:

$$
\frac{\partial u}{\partial t}=-\nabla^{2}\left(c\left(\left|\nabla^{2} u\right|\right) \nabla^{2} u\right)-\lambda \cdot\left(u-u^{0}\right)
$$

So we can discretize and iterate to solve the equation.

To solve the model in (4.5) efficiently and reliably, we propose a simple symmetric difference algorithm based on four neighboring systems (seen in Figure 2). 
We calculate Laplacian of the image intensity function as

$$
\left.\nabla^{2} u\right|_{i, j}=\frac{u_{i+1, j}+u_{i-1, j}+u_{i, j+1}+u_{i, j-1}-4 u_{i, j}}{h^{2}}
$$

where $h$ is space grid size. Given a time step $\Delta t$, (4.5) can be discretized as

$$
u^{n+1}=u^{n}-\Delta t\left(\nabla^{2}\left(c\left(\left|\nabla^{2} u^{n}\right|\right) \nabla^{2} u^{n}\right)+\lambda\left(u^{n}-u^{0}\right)\right) .
$$

Similar as [24], we define

$$
c(s)=\frac{1}{1+(s / k)^{2}}
$$

where $k$ is a parameter.

So the symmetric fourth-order PDE denoising algorithm is as follows.

Step 1. Initialization: select the constants $\lambda, k, h, \Delta t$ and choose an initial function (image) $u$.

Step 2. Compute $\nabla^{2} u$ and $\left|\nabla^{2} u\right|$ using (4.6).

Step 3. Compute $c\left(\left|\nabla^{2} u^{n}\right|\right)$ using (4.8).

Step 4. Update $u$ using (4.7).

Step 5. Repeat Steps 2 and 4 until convergence.

Figure 4 shows the results for a medical image with Gaussian white noise of mean 0 and variance 0.01 . Median filter is applied to alleviating the speckle effects in the processed image. We can see from Figure 4 that the new fourth-order PDE method obtains the biggest PSNR values in all filter method and can avoid the block effect in Figure 4(f). At the same time, the last result of the new method (Figure $4(\mathrm{j})$, PSNR $=28.4746 \mathrm{~dB}$ ) is better than the original fourth-order PDE method (Figure 4(b), PSNR = $27.7743 \mathrm{~dB}$ ) not only in PSNR values but also in visual quality.

\section{Multiplicative Noise Removal Algorithm Based on Fourth-Order PDE Model}

Objective of most traditional algorithms is to deal with additive noise, but the result is not ideal for the big multiplicative noise. This paper proposes a new multiplicative noise removal algorithm and combines the denoising algorithm with image frequency domain. The whole process is as follows.

Firstly, remove the additive noise $n$ in model (2.1) by denoising algorithm, then the model is simplified as

$$
u_{0}(x, y)=h_{d}(x, y) * f(x, y)
$$




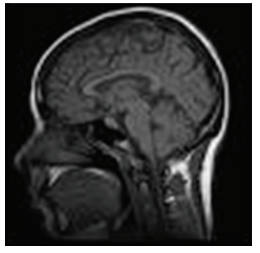

(a)

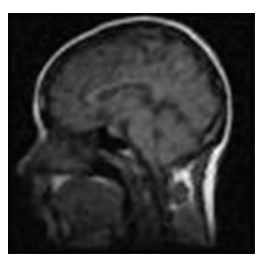

(f)

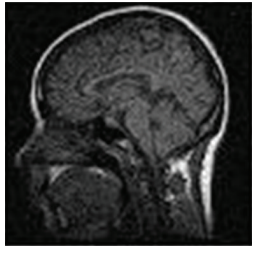

(b)

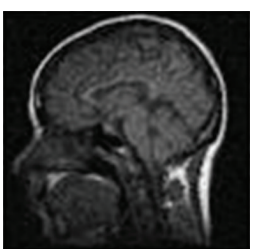

(g)

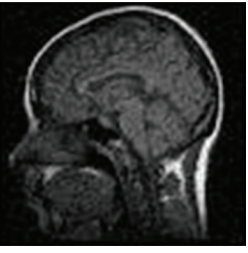

(c)

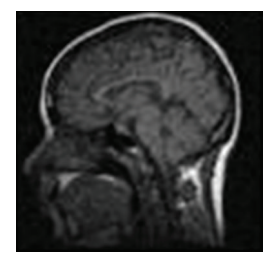

(h)

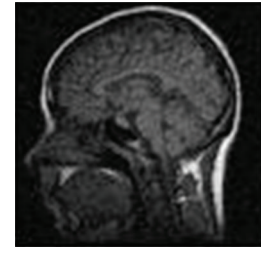

(d)

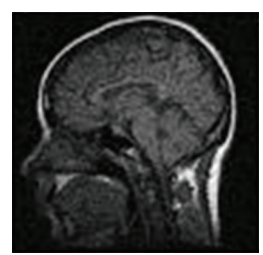

(i)

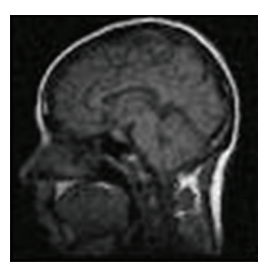

(e)

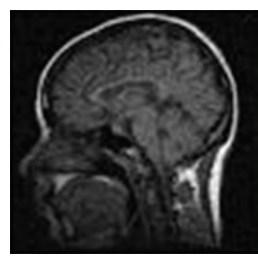

(j)

Figure 4: Detail of the denoising medical image with different methods. (a) A slice of an MRI brain image; (b) image with Gaussian white noise of mean 0 and variance 0.01, PSNR = 21.0980; (c) denoised with median filter (template: $3 \times 3$ ), PSNR = 27.3774; $(\mathrm{d})$ denoised with averaging filter (template: $3 \times 3$ ), PSNR $=27.7701$; $(\mathrm{e})$ denoised with Wiener filter $($ template: $5 \times 5)$, PSNR $=27.8061$; $(\mathrm{f})$ denoised with TVM $(\lambda=0.01,10$ iterations), PSNR = 24.9200; $(\mathrm{g})$ results with the original fourth-order PDE model, PSNR = 27.6730; $(\mathrm{h})$ denoised $(\mathrm{g})$ with Median filter, PSNR = 27.7743; (i) results with the new fourthorder PDE model, PSNR = 26.9902; (j) denoised (i) with median filter, PSNR = 28.4746.

Secondly, convolution in (5.1) changes to product according to fast Fourier transform (FFT):

$$
U(u, v)=H_{d}(u, v) \cdot F(u, v),
$$

where $U(u, v), H_{d}(u, v)$, and $F(u, v)$ denote FFT of $u_{0}(x, y), h_{d}(x, y)$, and $f(x, y)$, respectively.

Thirdly, (5.2) can be rewritten by logarithmic transformation (LN) as follows:

$$
\ln U(u, v)=\ln F(u, v)+\ln H_{d}(u, v) .
$$

Fourthly, $\ln H_{d}(u, v)$ in (5.3) can be regarded as additive noise in image frequency domain, and we can remove it by some additive denoising algorithms, such as TVM and fourth-order PDE model. Therefore,

$$
\ln U(u, v)=\ln F(u, v) .
$$

Fifthly, by exponential transform (EXP), (5.4) is rewritten as

$$
U(u, v)=F(u, v) .
$$

Sixthly, by inverse fast Fourier transform for (5.5), we can get

$$
u=f,
$$




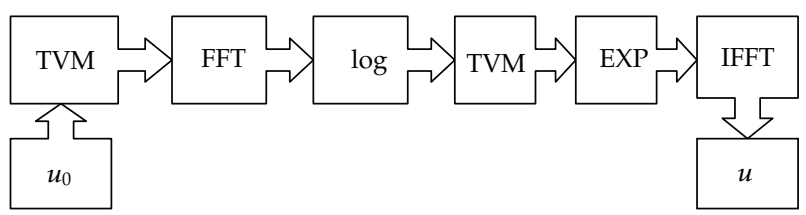

Figure 5: Structure of multiplicative noise removal algorithm based on the total variational model.

Table 1: PSNR values obtained with difference filters (template $3 \times 3$ ).

\begin{tabular}{lccccc}
\hline \multirow{2}{*}{ Noise level } & \multicolumn{4}{c}{ PSNR(dB) for Lena/vegetables image } & \\
& Noisy image & Average filter & Median filter & TV filter & MNRATV \\
\hline 0.05 & $13.73 / 13.67$ & $22.08 / 22.15$ & $23.66 / 23.56$ & $25.06 / 25.26$ & $\mathbf{2 5 . 4 7 / 2 6 . 0 4}$ \\
0.10 & $11.40 / 11.39$ & $19.71 / 19.74$ & $21.32 / 21.25$ & $22.48 / 22.48$ & $\mathbf{2 3 . 8 1 / 2 4 . 1 4}$ \\
0.15 & $10.25 / 1024$ & $18.36 / 18.46$ & $19.85 / 19.80$ & $20.64 / 20.74$ & $\mathbf{2 2 . 6 4 / 2 2 . 9 2}$ \\
0.20 & $9.54 / 9.52$ & $17.46 / 17.50$ & $18.78 / 18.66$ & $19.44 / 19.40$ & $\mathbf{2 1 . 7 4 / 2 1 . 7 8}$ \\
0.25 & $9.05 / 9.05$ & $16.84 / 16.87$ & $17.96 / 17.83$ & $18.54 / 18.56$ & $\mathbf{2 1 . 0 5 / 2 1 . 1 2}$ \\
0.30 & $8.69 / 8.68$ & $16.40 / 16.49$ & $17.29 / 17.16$ & $17.86 / 17.85$ & $\mathbf{2 0 . 4 6 / 2 0 . 5 1}$ \\
0.35 & $8.42 / 8.40$ & $16.02 / 15.98$ & $16.67 / 16.62$ & $17.29 / 17.33$ & $\mathbf{1 9 . 9 3 / 2 0 . 0 2}$ \\
0.40 & $8.19 / 8.20$ & $15.68 / 15.71$ & $16.24 / 16.40$ & $16.86 / 16.90$ & $\mathbf{1 9 . 5 6 / 1 9 . 6 2}$ \\
\hline
\end{tabular}

where $u$ in (5.6) is considered as the denoised image got by our algorithm. There are two denoising processes in the multiplicative noise removal framework, that is, the first step and the fourth step, if we select the denoising methods all as TVM removal framework of all as TVM, structure of multiplicative noise removal algorithm can be seen below.

In Figure 5, multiplicative noise removal algorithm considers the natural image noise as two parts, convolution changes to product by Fourier transform and product changes to summation by logarithm, then noise can be removal according to total variation model and the image is rebuilt.

\section{Experimental Results}

We use MATLAB 7.10 (R2010a) as the tool to carry out all algorithms a PC equipped with an Intel Core i3-2330 M CPU at $2.20 \mathrm{GHz}$ and 4G RAM memory and Windows 7 operating system. Denoising performance is evaluated using the PSNR (peak signal-to-noise ratio) in $\mathrm{dB}$ which defined by

$$
\operatorname{PSNR}=10 \cdot \log _{10} \frac{R^{2} M \cdot N}{[f(x, y)-u(x, y)]^{2}},
$$

where $u(x, y)$ denotes the restored image with respect to the original image $f(x, y), R=255$, and $M$ and $N$ are the wide and high of image.

The effectiveness of the new multiplicative noise removal algorithm is based on the total variation model (MNRATV) shown in Table 1, Figures 6 and 7. The sizes of the noisy "Lena" and "vegetables" images is all $512 \times 512$. The numerical results are listed in Table 1 and compared in Figure 6. Visual quality is shown in Figure 7. Experimental results show that 


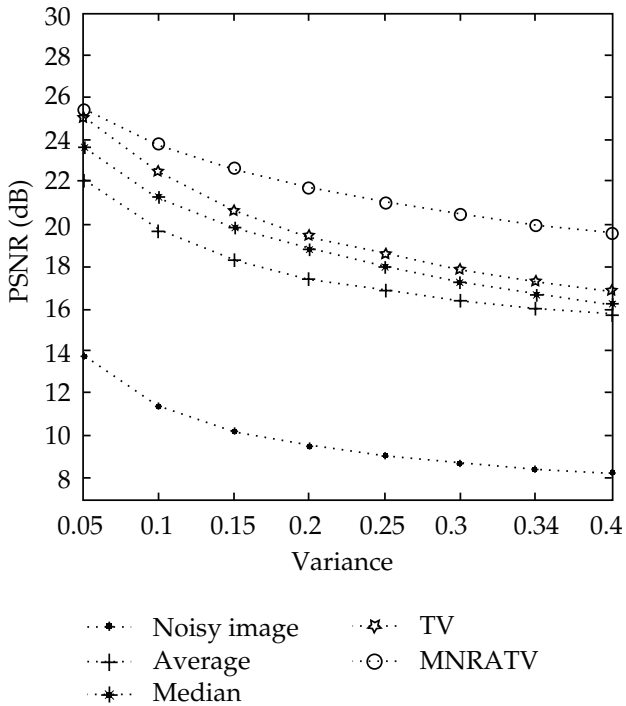

(a)

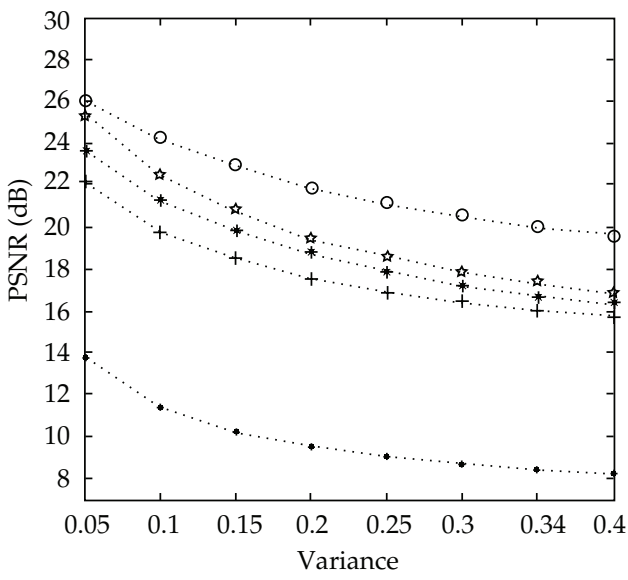

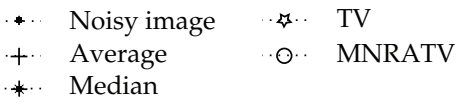

(b)

Figure 6: PSNR values in Table 1 plotted together. (a) Lena image; (b) vegetables image.

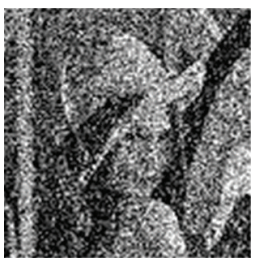

(a)

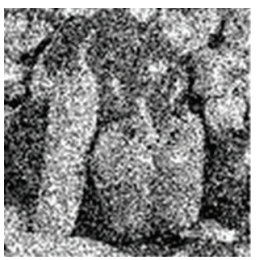

(f)

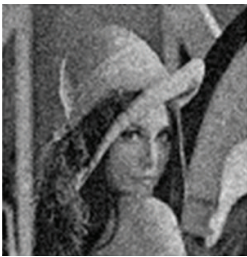

(b)

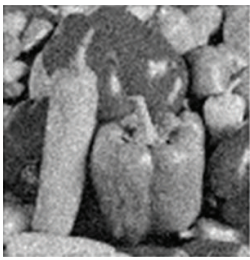

(g)

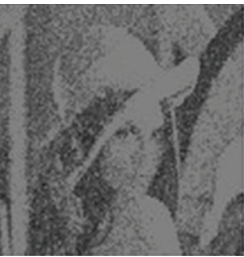

(c)

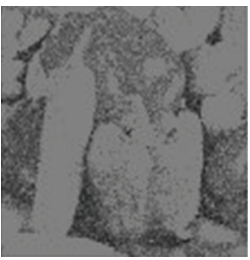

(h)

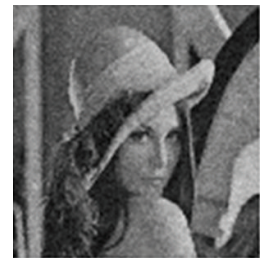

(d)

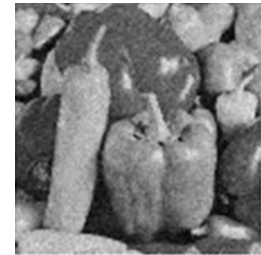

(i)

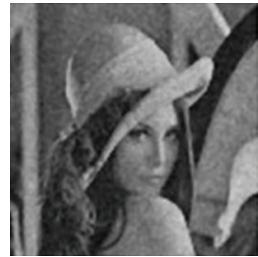

(e)

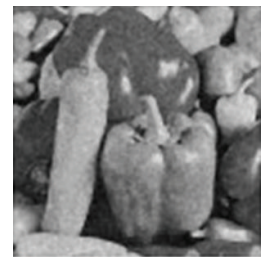

(j)

Figure 7: Results of difference noise removal algorithms for noisy "Lena" and "vegetables" images. (a) The multiplicative noisy image; (b) results of Median filter algorithm; (c) results of Averaging filter algorithm; (d) results of TVM; (e) results of MNRATV.

the new method is available. It is better than the traditional denoising algorithm not only in PSNR values but also in visual quality.

We can see from Table 1 and Figure 6 that the PSNR values of the restored images by MNRATV are higher than restored images by all the other methods. It is little bigger than those by TVM when the noise level is lower. The results of MNRATV and TVM are shown in Figure 8 . 


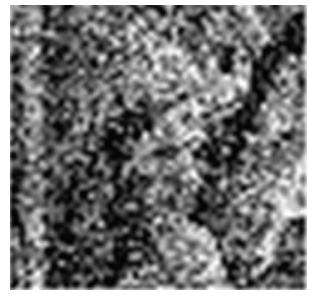

var $=0.05$

(a)

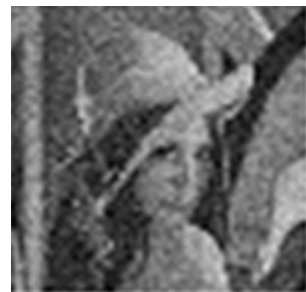

$\mathrm{PSNR}=22.45 \mathrm{~dB}$

(e)

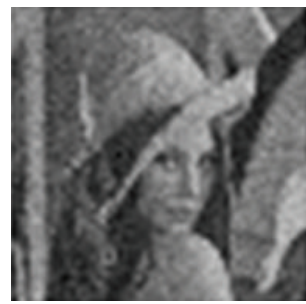

$\mathrm{PSNR}=22.62 \mathrm{~dB}$

(i)

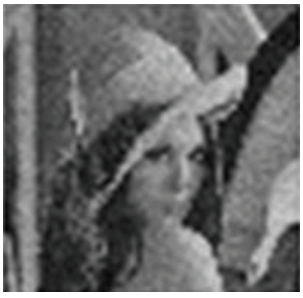

$\mathrm{PSNR}=25.07 \mathrm{~dB}$

(b)

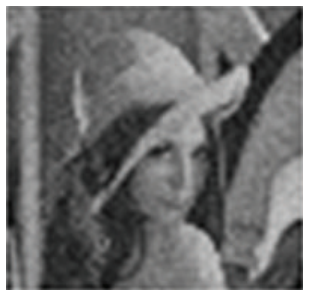

PSNR $=23.9 \mathrm{~dB}$

(f)

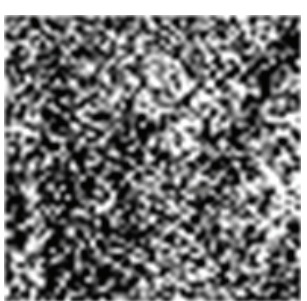

$\operatorname{var}=0.2$

(j)

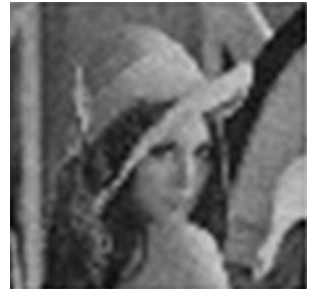

$\mathrm{PSNR}=25.47 \mathrm{~dB}$

(c)

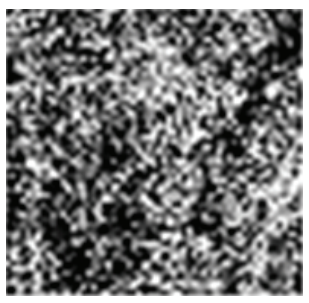

$\operatorname{var}=0.15$

$(\mathrm{g})$

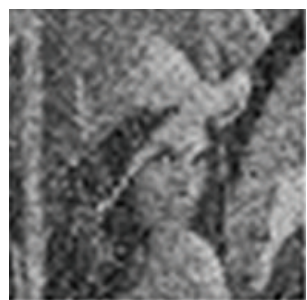

$\mathrm{PSNR}=19.48 \mathrm{~dB}$

(k)

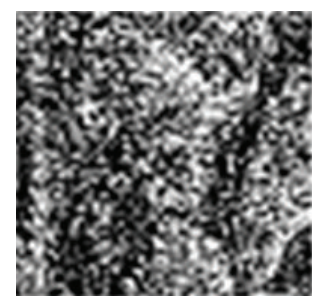

var $=0.1$

(d)

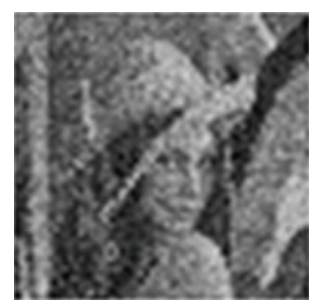

PSNR $=20.63 \mathrm{~dB}$

(h)

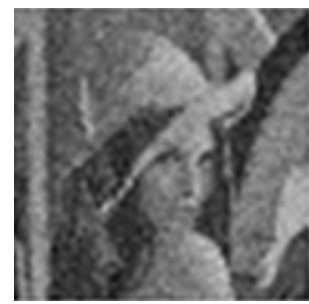

$\mathrm{PSNR}=21.78 \mathrm{~dB}$

(1)

Figure 8: Results of MNRATV and TVM. Column 1 is the multiplicative noise with variance 0.05, 0.10, 0.15 , and 0.20; respectively, Column 2 is the corresponding results with TVM, and Column 3 is the corresponding results with MNRATV.

Table 2: Different multiplicative noise removal method constructed with exist model.

\begin{tabular}{lcc}
\hline Denoising method 1 & Denoising method 2 & New method \\
\hline TVM & TVM & MNRA1(MNRATV) \\
FPDE & TVM & MNRA2 \\
TVM & FPDE & MNRA3 \\
FPDE & FPDE & MNRA4 \\
\hline
\end{tabular}

There are two denoising methods in the first step and fourth step of the multiplicative noise removal framework; we can call it denoising method 1 and denoising method 2 . If they are all chosen as TVM, then the whole framework in Figure 5 is called MNRA1 method, which is called MNRATV before. TVM and fourth-order PDE (FPDE) model which we introduced in Section 4 constitutes four methods. Details are shown in Table 2.

Different methods are employed to remove the noisy Lena image with different variance. PSNR values are shown in Table 3. Seen from Table 3, TVM or FPDE directly is not 
Table 3: PSNR values obtained with difference algorithm for Lena image.

\begin{tabular}{lccccccc}
\hline \multirow{2}{*}{ variance } & Noisy image & TVM & FPDE & MNRA1 & MNRA2 & MNRA3 & MNRA4 \\
\hline 0.05 & 13.6935 & 25.0739 & 24.0739 & 25.4833 & 25.6347 & $\mathbf{2 5 . 8 1 9 0}$ & 25.7443 \\
0.10 & 11.4336 & 22.4860 & 22.3232 & 23.9355 & $\mathbf{2 4 . 2 3 8 2}$ & 23.8612 & 23.9770 \\
0.15 & 10.2712 & 20.7197 & 20.7700 & 22.7277 & $\mathbf{2 3 . 2 1 0 1}$ & 22.4985 & 22.7830 \\
0.20 & 9.5307 & 19.4144 & 19.6830 & 21.6790 & $\mathbf{2 2 . 4 6 6 7}$ & 21.3851 & 21.7750 \\
0.25 & 9.0769 & 18.6046 & 18.8871 & 21.0824 & $\mathbf{2 1 . 8 9 2 7}$ & 20.6409 & 21.0582 \\
0.30 & 8.6769 & 17.7913 & 18.0713 & 20.3577 & $\mathbf{2 1 . 2 6 1 6}$ & 19.9417 & 20.3536 \\
0.35 & 8.4000 & 17.2305 & 17.4785 & 19.8687 & $\mathbf{2 0 . 7 3 7 7}$ & 19.3328 & 19.7712 \\
0.40 & 8.1948 & 16.8051 & 16.9839 & 19.5339 & $\mathbf{2 0 . 3 1 3 5}$ & 19.1239 & 19.4284 \\
\hline
\end{tabular}

good because of the multiplicative noise type. Median filter cannot be exploited as denoising method 2 since complex number is generated by the Fourier transform. Denoising method 1 is selected as FPDE in MNRA2, and it gets better results.

\section{Conclusion}

PDE models have been widely applied in image processing community especially in image denoising. However, traditional PDE-based methods have some drawbacks unless the governing equations are both incorporating appropriate parameters and discretized by suitable numerical schemes. In this paper, a new fourth-order PDE model is introduced by improving the original fourth-order one [24] in order to avoid the blocky effect. To solve the model efficiently and reliably, we suggest a symmetrically difference schemes. Median filtering is exploited to alleviate the speckle effects in the processed image in succession. Accordingly, a new multiplicative noise removal algorithm based on the proposed fourth-order PDE model is presented. To remove the multiplicative noise, the convolution is changed into a product by applying the Fourier transform. Furthermore, the multiplicative noise is converted into the additive one by using a logarithmic transformation. Then the noise can be removed by applying the proposed PDE model. Experimental results have shown the effectiveness of the proposal.

\section{Acknowledgments}

This paper is partially supported by NSFC $(61070087,61105130)$, the Natural Science Foundation of Guangdong Province (S2011040000433, S2011040004017), the Science \& Technology Planning Project of Shenzhen City (JC200903130300A, JYC200903250175A), and the Opening Project of Guangdong Province Key Laboratory of Computational Science of Sun Yat-Sen University (201106002) and Teaching Reform and Research Project for Young Teachers of Shenzhen University (JG2010118). The authors would like to thank the Key Laboratory of Medical Image Processing in Southern Medical University for providing original medical images. 


\section{References}

[1] Q. Y. Ruan and Y. Z. Ruan, Digital Image Processing, Publishing House of Electronics Industry, Beijing, China, 2nd edition, 2004.

[2] Q. H. Chang and T. Yang, "A lattice Boltzmann method for image denoising," IEEE Transactions on Image Processing, vol. 18, no. 12, pp. 2797-2802, 2009.

[3] C. Chaux, L. Duval, A. Benazza-Benyahia, and J.-C. Pesquet, "A nonlinear Stein-based estimator for multichannel image denoising," IEEE Transactions on Signal Processing, vol. 56, no. 8, part 2, pp. 38553870, 2008.

[4] P. Perona and J. Malik, "Scale-space and edge detection using anisotropic diffusion," IEEE Transactions on Pattern Analysis and Machine Intelligence, vol. 12, no. 7, pp. 629-639, 1990.

[5] S. Osher and J. A. Sethian, "Fronts propagating with curvature-dependent speed: algorithms based on Hamilton-Jacobi formulations," Journal of Computational Physics, vol. 79, no. 1, pp. 12-49, 1988.

[6] S. Osher and C. W. Shu, "High-order essentially nonoscillatory schemes for Hamilton-Jacobi equations," SIAM Journal on Numerical Analysis, vol. 28, no. 4, pp. 907-922, 1991.

[7] B. Chen, Y. Li, and J.-L. Cai, "Noisy image segmentation based on nonlinear diffusion equation model," Applied Mathematical Modelling, vol. 36, no. 3, pp. 1197-1208, 2012.

[8] Z. Liao, S. Hu, and W. Chen, "Determining neighborhoods of image pixels automatically for adaptive image denoising using nonlinear time series analysis," Mathematical Problems in Engineering, vol. 2010, Article ID 914564, 14 pages, 2010.

[9] S. Hu, Z. Liao, D. Sun, and W. Chen, "A numerical method for preserving curve edges in nonlinear anisotropic smoothing," Mathematical Problems in Engineering, vol. 2011, Article ID 186507, 14 pages, 2011.

[10] M. Li, "Fractal time series-a tutorial review," Mathematical Problems in Engineering, vol. 2010, Article ID 157264, 26 pages, 2010.

[11] Z. Shang, L. Zhang, S. Ma, B. Fang, and T. Zhang, "Incomplete time series prediction using maxmargin classification of data with absent features," Mathematical Problems in Engineering, vol. 2010, 14 pages, 2010.

[12] M. Freiberger, H. Egger, and H. Scharfetter, "Nonlinear inversion schemes for fluorescence optical tomography," IEEE Transactions on Biomedical Engineering, vol. 57, no. 11, pp. 2723-2729, 2010.

[13] Z. Liao, S. Hu, D. Sun, and W. Chen, "Enclosed Laplacian operator of nonlinear anisotropic diffusion to preserve singularities and delete isolated points in image smoothing," Mathematical Problems in Engineering, vol. 2011, Article ID 749456, 15 pages, 2011.

[14] M. Li and W. Zhao, "Visiting power laws in cyber-physical networking systems," Mathematical Problems in Engineering, vol. 2012, Article ID 302786, 13 pages, 2012.

[15] M. Li, C. Cattani, and S. Chen, "Viewing sea level by a one-dimensional random function with long memory," Mathematical Problems in Engineering, vol. 2011, Article ID 654284, 13 pages, 2011.

[16] F. Zhang, Y. M. Yoo, L. M. Koh, and Y. Kim, "Nonlinear diffusion in laplacian pyramid domain for ultrasonic speckle reduction," IEEE Transactions on Medical Imaging, vol. 26, no. 2, pp. 200-211, 2007.

[17] M. Ceccarelli, V. De Simone, and A. Murli, "Well-posed anisotropic diffusion for image denoising," IEE Proceedings: Vision, Image and Signal Processing, vol. 149, no. 4, pp. 244-252, 2002.

[18] M. Li, M. Scalia, and C. Toma, "Nonlinear time series: computations and applications," Mathematical Problems in Engineering, vol. 2010, Article ID 101523, 5 pages, 2010.

[19] L. X. Shen, M. Papadakis, I. A. Kakadiaris et al., "Image denoising using a tight frame," IEEE Transaction on Image Processing, vol. 15, no. 5, pp. 1254-1263, 2006.

[20] B. Chen and W.-S. Chen, "Noisy image segmentation based on wavelet transform and active contour model," Applicable Analysis, vol. 90, no. 8, pp. 1243-1255, 2011.

[21] T. Celik and K. Ma, "multitemporal image change detection using undecimated discrete wavelet transform and active contours," IEEE Transactions on Geoscience and Remote Sensing, vol. 49, no. 2, pp. 706-716, 2011.

[22] T. Celik and K. Ma, "Unsupervised change detection for satellite images using dual-tree complex wavelet transform," IEEE Transactions on Geoscience and Remote Sensing, vol. 48, no. 3, pp. 1199-1210, 2010.

[23] S. Kim, "PDE-based image restoration: a hybrid model and color image denoising," IEEE Transaction on Image Processing, vol. 15, no. 5, pp. 1163-1170, 2006. 
[24] Y.-L. You and M. Kaveh, "Fourth-order partial differential equations for noise removal," IEEE Transactions on Image Processing, vol. 9, no. 10, pp. 1723-1730, 2000.

[25] B. Chen, W.-S. Chen, L.-W. Zhang et al., "Image restoration based on fourth-order PDE model," in Proceedings of the IEEE International Conference on Natural Computation, vol. 5, pp. 549-554, Jinan, China, 2008.

[26] B. Chen, P.-C. Yuen, J.-H. Lai, and W.-S. Chen, "Image segmentation and selective smoothing based on variational framework," Journal of Signal Processing Systems, vol. 54, pp. 145-158, 2009.

[27] L. Rudin, S. Osher, and E. Fatemi, "Nonlinear total variation based noise removel algorithms," Physica D, vol. 60, pp. 259-268, 1992.

[28] S. D. Babacan, R. Molina, and A. Katsaggelos, "Variational bayesian blind deconvolution using a total variation prior," IEEE Transactions on Image Processing, vol. 18, no. 1, pp. 12-26, 2009.

[29] L. Rudin and S. Osher, "Total variation based image restoration with free local constraints," in Proceedings of the 1st IEEE International Conference on Image Processing, vol. 1, pp. 31-35, 1994.

[30] T. F. Chan, S. Osher, and J. H. Shen, "The digital TV filter and Nonlinear Denoising," IEEE Transaction on Image Processing, vol. 10, no. 2, pp. 231-241, 2001.

[31] A. Beck and M. Teboulle, "Fast gradient-based algorithms for constrained total variation image denoising and deblurring problems," IEEE Transactions on Image Processing, vol. 18, no. 11, pp. 24192434, 2009.

[32] C. Drapaca, "A nonlinear total variation-based denoising method with two regularization parameters," IEEE Transactions on Biomedical Engineering, vol. 56, no. 3, pp. 582-586, 2009.

[33] M. Kumar and S. Dass, "A total variation-based algorithm for pixel-level image fusion," IEEE Transactions on Image Processing, vol. 18, no. 9, pp. 2137-2143, 2009.

[34] P. Rodríguez and B. Wohlberg, "Efficient minimization method for a generalized total variation functional," IEEE Transactions on Image Processing, vol. 18, no. 2, pp. 322-332, 2009.

[35] T. Zeng and M. Ng, "On the total variation dictionary model," IEEE Transactions on Image Processing, vol. 19, no. 3, pp. 821-825, 2010.

[36] B. C. Vemuri, M. Liu, S. Amari et al., "Total bregman divergence and its applications to DTI analysis," IEEE Transactions on Medical Imaging, vol. 30, no. 2, pp. 475-483, 2011.

[37] S. Ramani, P. Thevenaz, and M. Unser, "Regularized interpolation for noisy images," IEEE Transactions on Medical Imaging, vol. 29, no. 2, pp. 543-558, 2010.

[38] J. Yao, J. Chen, and C. Chow, "Breast tumor analysis in dynamic contrast enhanced MRI using texture features and wavelet transform," IEEE Journal of Selected Topics in Signal Processing, vol. 3, no. 1, pp. 94-100, 2009.

[39] M. Zibetti and A. R. De Pierro, "A new distortion model for strong inhomogeneity problems in EchoPlanar MRI," IEEE Transactions on Medical Imaging, vol. 28, no. 11, pp. 1736-1753, 2009.

[40] J. M. Bioucas-Dias and M. A. T. Figueiredo, "Multiplicative noise removal using variable splitting and constrained optimization," IEEE Transactions on Image Processing, vol. 19, no. 7, pp. 1720-1730, 2010.

[41] L. Rudin, P. Lions, and S. Osher, "Multiplicative denoising and deblurring: theory and algorithms," in Geometric Level Set Methods in Imaging, Vision, and Graphics, S. Osher and N. Paragios, Eds., pp. 103-120, Springer, New York, NY, USA, 2003.

[42] J. Goodman, "Some fundamental properties of speckle," Journal of the Optical Society of America, vol. 66, pp. 1145-1150, 1976.

[43] Y. Yu and S. T. Acton, "Speckle reducing anisotropic diffusion," IEEE Transactions on Image Processing, vol. 11, no. 11, pp. 1260-1270, 2002.

[44] K. Krissian, C.-F. Westin, R. Kikinis, and K. G. Vosburgh, “Oriented speckle reducing anisotropic diffusion," IEEE Transactions on Image Processing, vol. 16, no. 5, pp. 1412-1424, 2007. 


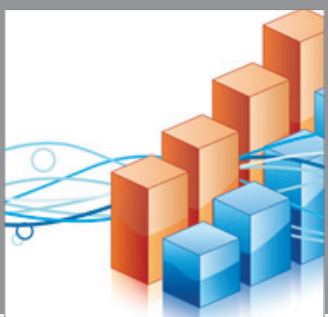

Advances in

Operations Research

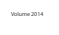

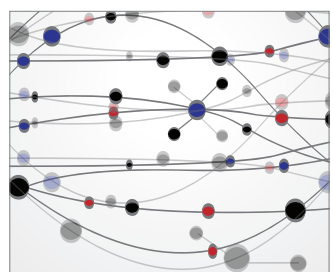

\section{The Scientific} World Journal
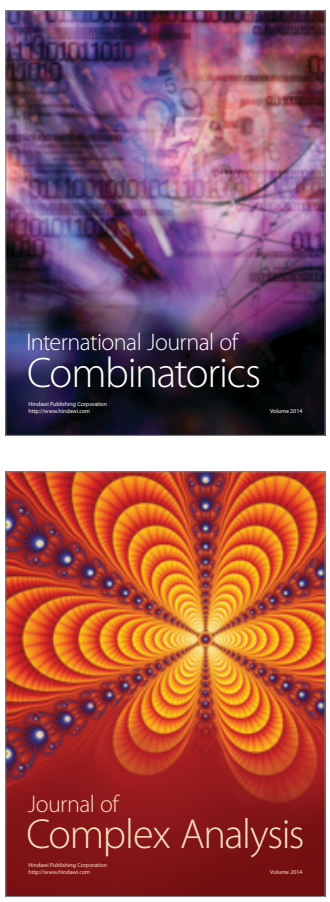

International Journal of

Mathematics and

Mathematical

Sciences
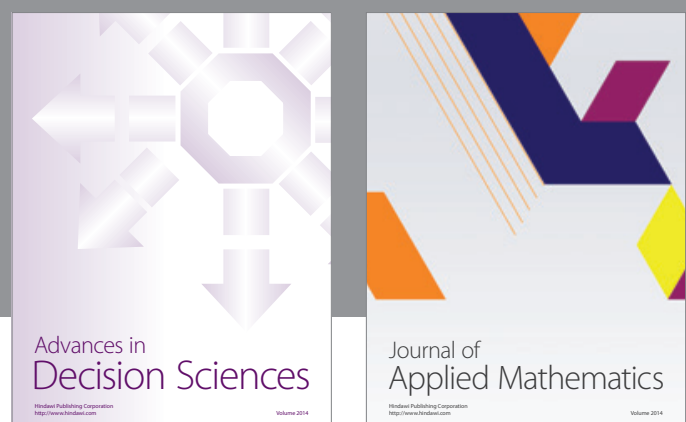

Journal of

Applied Mathematics
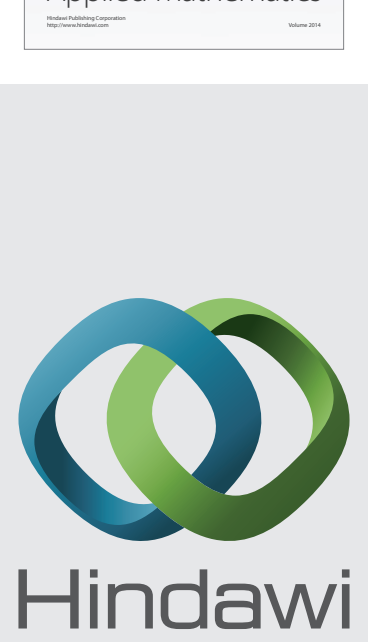

Submit your manuscripts at http://www.hindawi.com
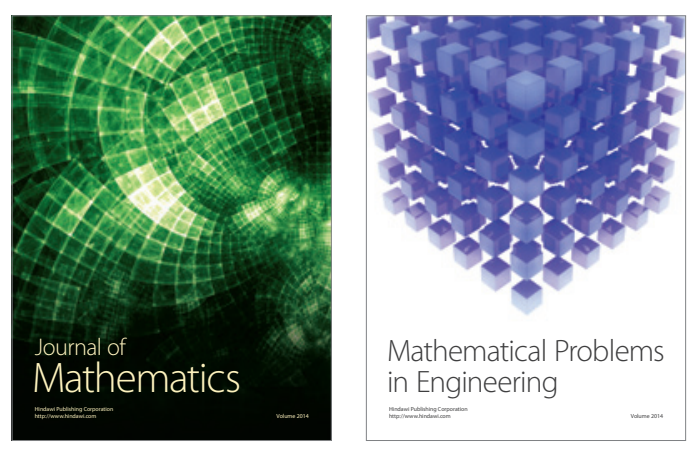

Mathematical Problems in Engineering
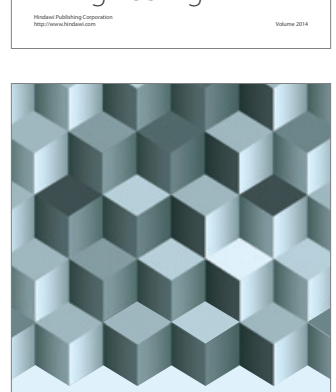

Journal of

Function Spaces
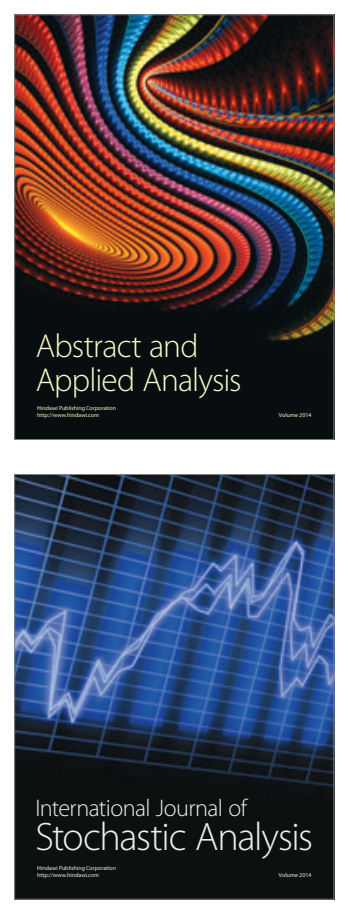

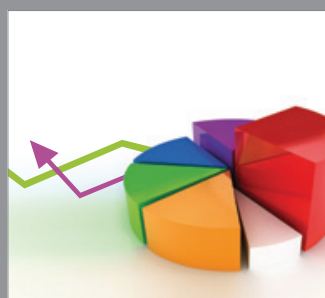

ournal of

Probability and Statistics

Promensencen
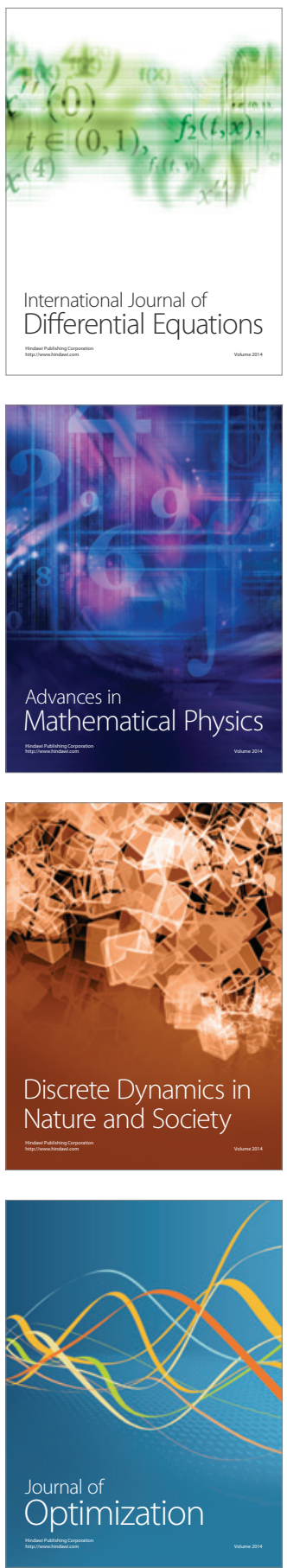